\title{
Concentración regional de la población por niveles de riqueza hídrica en Colombia*
}

\section{Regional concentration of the population wealth water levels in Colombia}

Gustavo Correa Assmus**

Recibido: 27 de octubre de 2014

Revisado: 14 de noviembre de 2014

Aprobado: 16 de marzo de 2015

\section{Resumen}

Diferentes manifestaciones sociales, económicas y ambientales han modificado los intereses de acceso al agua en Colombia, por ello, el objetivo del presente artículo pretende establecer el grado de relación entre la concentración poblacional y la riqueza de la oferta natural de agua en las cinco regiones naturales de Colombia. La metodología y el método toman en cuenta las siguientes acciones: 1) compilar estadísticas sobre la oferta hídrica y el crecimiento poblacional y 2) formular matemáticamente la distribución hídrica, la distribución poblacional y el índice de concentración, lo cual permite establecer sesgos regionales de carácter social y ambiental de manera sinérgica. Los resultados evidencian la pérdida de primacía del agua frente a lo económico en términos de propiciar

* Como citar este articulo: Correa, G. (2014). Concentración regional de la población por niveles de riqueza hídrica en Colombia. Revista CIFE, 16(25), 153-165

** Programa doctoral en Agrociencias, Universidad de La Salle, Bogotá, Colombia. gcorrea@unisalle.edu.co. 
la concentración poblacional regional. Este fenómeno se manifiesta en la región Andina de Colombia, donde se encuentra la mayor concentración poblacional, la menor oferta hídrica y, por lo tanto, la mayor vulnerabilidad frente al abastecimiento hídrico; le sigue la región Caribe y luego las demás regiones estudiadas.

Palabras clave: agua, concentración, regiones.

Clasificación JEL: Q01, Q25; Q32; Q57

\begin{abstract}
Different social, economic and environmental events have changed the interests of access to water in Colombia, therefore, the objective of this article seeks to establish the degree of relationship between population density and richness of the natural supply of water in the five natural regions from Colombia. The methodology and the method takes into account the following: 1) compile statistics on water supply and population growth and 2) mathematically formulate water distribution, population distribution and the concentration index, which allows for regional biases of character social and environmental synergistically. The results show the loss of primacy of water against the economic in terms of promoting the regional population concentration. This phenomenon is manifested in the Andean region of Colombia, where the greatest population concentration, the lower water supply and, therefore, greater vulnerability to water supply; followed by the Caribbean region and then other regions studied.
\end{abstract}

Keywords: Water, concentration regions.

Classification JEL: Q01, Q25; Q32; Q57 


\section{Introducción}

Colombia posee un importante y reconocido acervo hídrico, el cual permite contar con una oferta per cápita anual de $50000 \mathrm{~m}^{3}$ habitante $^{-1}$ (IDEAM, 2010). Sin embargo, esta riqueza natural se distribuye de manera desigual por el territorio, evento que, junto con los fenómenos de El Niño, La Niña y el cambio climático interrogan sobre posibles variaciones en la distribución poblacional como respuesta a su necesidad de agua. En este sentido, la literatura es abundante en la búsqueda de procesos de adaptación social ante el cambio climático por organizaciones como ONU-Hábitat, el Banco Mundial y el BID, entre otras. De manera complementaria, cabe tomar en cuenta que la riqueza hídrica nacional, en términos de su rendimiento, es seis veces superior al promedio mundial $\left(10 \mathrm{~L} \mathrm{~s}^{-1} \mathrm{~km}^{-2}\right)$ y tres veces superior al rendimiento de Latinoamérica $\left(21 \mathrm{~L} \mathrm{~s}^{-1} \mathrm{~km}^{-2}\right)$, esto de acuerdo con el Estudio nacional de aguas (IDEAM, 2010). Esta oferta es poco entendida en sus ciclos, distribución y tenencia, tanto por los órdenes gubernamentales como por los sociales, lo cual propicia desinterés en torno a su conservación y preservación, fenomenología característica del comportamiento social frente a los bienes comunes o libres ampliamente analizada por Olson (1985), Hardin (1995) y Ostrom (2009).

A su vez, las regiones naturales del país son producto de relaciones históricas de carácter socionatural que, a través del tiempo, han definido particularidades económicas y culturales que diferencian a las regiones Andina, Caribe, Pacífica, la Orinoquía y la Amazonía (IGAC, 2011). La concentración poblacional, como respuesta a los intereses para acceder a la oferta regional de agua, ha reconocido a lo largo de la historia diferentes clasificaciones urbanas, como la región plan, el epicentro regional, la región cultural, la regionalización nodal o la ciudad región, como lo describe la CEPAL (2009) para América Latina. Históricamente, las autonomías regionales fragmentaron el territorio nacional, situación que no permitió una primacía urbana, pero se facilitó el empoderamiento de un núcleo urbano de relativa importancia para cada región, sustentado por las condiciones climáticas, topográficas, la expansión de la frontera agrícola y la especialización productiva, origen de la estructura multipolar nacional. No se quiere desconocer con ello la primacía de Bogotá a nivel nacional, dados su desarrollo y oportunidades como capital cosmopolita, las cuales atraen buena parte de la migración interna (CEPAL, 2009) y la movilidad por efecto de las violencias.

Como Botiva, Groot, Herrera y Mora (1989), Rojas (2010) y Correa (2011) lo plantean, la desigual concentración poblacional a orillas de los ríos y lagos definió, desde la época prehispánica y posteriormente en la Colonia, una dispar tenencia del agua en la población asentada en las regiones naturales de Colombia: Andina, Caribe, Pacífica, Orinoquía y Amazonía. Sin embargo, la historia no se presenta concluyente en la tendencia hacia la concentración poblacional con base en la distribución de la riqueza hídrica. Actualmente, bajo el influjo climático, el desarrollo socioeconómico y la migración 
rural, presionada por el conflicto armado interno de acuerdo con Guevara (2012), arrojan poca claridad en torno a la concentración poblacional de base hídrica. Por ello, las concentraciones poblacionales en las regiones pueden obedecer a condiciones de primacía hídrica, así como pueden no serlo, atendiendo a una mezcla de intereses en la que participan la necesidad hídrica junto con expectativas económicas y sociales que, en últimas, determinan el grado de desarrollo interregional.

Según Henderson (2000), la concentración urbana es una expresión de la desigualdad territorial. En términos del acceso al recurso agua, se traduce en concentraciones poblacionales agenciadas por las facilidades propias del desarrollo urbano frente al rural, junto con la presencia de sesgos geográficos en el asentamiento humano y presiones significativas sobre la oferta hídrica regional, de conformidad con los argumentos sobre asentamientos localizados de Krugman (1991) y CEPAL (2009). Por su parte, Montenegro (1996) considera a Colombia el país más desconcentrado (multipolar) espacialmente de América Latina debido a su diversidad geográfica regional y a la distribución de la actividad económica, articuladas con la presencia de diversos conglomerados urbanos principales (Bogotá, Medellín, Cali, Barranquilla y Bucaramanga) y secundarios (Cartagena, Villavicencio, Popayán, Manizales, Cúcuta, Pereira, Montería, Pasto e Ibagué).

En términos de Lombardo (2007) los paradigmas urbanos toman en consideración los fenómenos de concentración económica, de gestión poblacional y de gobernabilidad de las regiones; sin embargo, la presencia de una red urbana ampliada con ciudades primarias y secundarias (caso Colombia) requiere de una mayor inversión, infraestructuras, conectividad y aporte económico para su desarrollo en detrimento de la ruralidad, condición de inequidad tendiente a subsanarse con acciones preferiblemente compensatorias no estructurales (Cuervo, 2004). Por ello, los indicadores de acceso al agua pueden resultar favorables en el ámbito urbano, no así las condiciones de acceso, la calidad del recurso, la disposición de efluentes y la contaminación, en general, en el contexto rural.

En este orden de ideas, se puede argumentar que las facilidades y oportunidades para el acceso al agua dinamizan la concentración poblacional de tipo regional. Por esta razón, es necesario tomar en consideración las condiciones naturales de las regiones y las manifestaciones del cambio climático partícipes en la disponibilidad de agua, lo cual genera efectos sobre la seguridad alimentaria, el costo de vida, la pobreza y el desarrollo en general. En este sentido, la ONU (2012) sustenta que las conexiones entre la urbanización y el crecimiento económico agencian condiciones de pobreza urbana y rural en las regiones; desde el acceso al agua, estas conexiones establecen asimetrías socioeconómicas en los asentamientos poblacionales informales y contribuyen a consolidar y reproducir la pobreza tanto urbana como rural. 
En este punto, la planeación ambiental nacional y regional de Colombia cobra relevancia, planeación que tradicionalmente no ha sido un ejercicio sistemático ni riguroso, sino más bien circunstancial, tanto en su promulgación como en su aplicación y control, lo cual genera debilidad en la preservación, la conservación y el manejo del recurso hídrico, y abre brechas para usos y aprovechamientos insostenibles, cuyo costo social y ambiental afecta principalmente a las comunidades más vulnerables, como lo sostiene Sánchez-Triana (2007). La concentración poblacional regional en torno a la riqueza hídrica de la nación es, en la mayoría de los casos, un proceso voluntario marginado del análisis y la caracterización de los rasgos dominantes sobre su convergencia, a fin de establecer decisiones de política hídrica equilibradas entre la protección del recurso agua, su oferta para un consumo que permita asegurar el desarrollo ambiental, socioeconómico y una calidad de vida digna frente a los impactos por escasez natural o estacional en las regiones (ONU-HÁBITAT, 2012).

Por lo anterior, el objeto de análisis se enfoca en dos perspectivas no excluyentes y relacionadas. La primera obedece a la diferencia en los niveles de riqueza hídrica regional a partir de la oferta natural de agua per cápita, y la segunda contempla la concentración poblacional en torno a esa riqueza. Con ello se pretende interpretar una concentración poblacional mediada, en primera instancia, por aspectos socioeconómicos e infraestructurales, seguida por el interés en la facilidad urbana para acceder al agua, lo cual conduce a suponer que la riqueza hídrica regional no es el elemento primordial para consolidar el asentamiento poblacional regional en Colombia.

\section{Aproximación metodológica}

Para el caso colombiano, las evidencias indican que la población asentada en la ruralidad dispersa es menor a la concentrada en las ciudades (DANE, 2005). A su vez, la teoría sostiene que la movilidad poblacional tiende a establecerse en regiones con ventajas comparativas frente a otras, en términos de facilitar mayores y mejores condiciones de vida (PNUD, 2009; GEPAL, 2012).

Desde esta perspectiva, y a fin de evaluar la relación entre la concentración poblacional y la oferta natural de agua, se recopiló información secundaria de entidades como la Comisión Económica para América Latina y el Caribe (CEPAL, Anuarios Estadísticos), el Programa de las Naciones Unidas para el Desarrollo (PNUD), el Programa de las Naciones Unidas para el Medio Ambiente (PNUMA, Informes GEO), el Programa de las Naciones Unidas para los Asentamientos Humanos (ONU-Hábitat), el Departamento Nacional de Estadísticas (DANE), el Departamento Nacional de Planeación (DNP), la Defensoría del Pueblo, el Instituto Geográfico Agustín Codazzi (IGAC) y el Instituto de Hidrología, Meteorología y Estudios Ambientales (IDEAM). 
Para el caso, se asumió la oferta hídrica per cápita como desagregado de la oferta hídrica nacional y la evolución poblacional entre los años 2000 a 2012. Este periodo es particularmente interesante debido a la presencia de impactos importantes de la pluviometría regional intensificada por el cambio climático, especialmente durante 2006, 2008 y 2010, con pérdidas económicas, físicas y humanas para el país (Defensoría del Pueblo, 2011). Además, la interpretación de resultados para este periodo (2000-2012) se considera de largo plazo, pues permite observar el comportamiento de las condiciones hídricas y poblacionales, así como establecer tendencias y comparaciones para las regiones naturales de Colombia con sus respectivos departamentos: 1) región Andina: Antioquia, Boyacá, Caldas, Cundinamarca, Huila, Norte de Santander, Quindío, Risaralda, Santander y Tolima; 2) región Caribe: departamentos de Atlántico, Bolívar, Cesar, Córdoba, La Guajira, Magdalena, San Andrés y Providencia y Sucre; 3) región Pacífica: Cauca, Chocó, Nariño y Valle del Cauca; 4) región Orinoquía: Arauca, Casanare, Meta y Vichada; 5) región Amazonía: Amazonas, Gaquetá, Guainía, Guaviare, Putumayo y Vaupés (IGAC, 2011).

El desarrollo del modelo se hace sobre la base de dos distribuciones de frecuencia (DHT y DPH) que conducen a una distribución normal con alta concentración de los casos en el promedio muestral. Complementariamente, con base en los resultados se desarrollan algunos indicadores que permiten consolidar las observaciones generales.

Para cada una de las distribuciones se estableció la siguiente formulación:

$\mathrm{DHT}=(\mathrm{Ln}$ Ohr $/$ Ln Ohn $)$, distribución hídrica regional.

$\mathrm{DPH}=(\mathrm{Ln}$ Ohr / Ln Ohn $)(\mathrm{Pr} / \mathrm{Pn})$, distribución poblacional de base hídrica.

La formulación fue construida a partir de una relación logarítmica entre las ofertas naturales de agua regional $(\mathrm{Ohr})$ y nacional $(\mathrm{Ohn})$, ponderada por la población asentada en cada una de las regiones $(\mathrm{Pr})$ y a nivel nacional $(\mathrm{Pn})$.

El grado de convergencia de la DPH se calcula a partir del coeficiente sigma $(\zeta)$, igual a la desviación estándar ( $\sigma$ ) de los logaritmos de la oferta hídrica regional $(\mathrm{Ohr})$. Luego, se establece el coeficiente de variación $(\mathrm{CV})$, el cual corresponde a sigma dividido por su media, lo anterior con el fin de plantear el coeficiente de convergencia, igual a:

$$
\mathrm{CG}=\mathrm{CV}(\mathrm{Pr} / \mathrm{Pn})
$$

Como indicadores complementarios para robustecer el análisis se tienen la participación regional poblacional (PRP), la densidad poblacional (DP), el índice de desarrollo humano (IDH), el acceso urbano al agua (AU), el acceso rural al agua (AR), la conexión domiciliaria urbana (CDU), el índice de riesgo al consumir por agua tratada (IRCA) y el índice de riesgo al consumir por agua no tratada (IRCAN). 


\section{Resultados}

La región Andina es la más densamente poblada (tabla 1), con un IDH alto, el IRCA más bajo del país debido al desarrollo en la infraestructura de saneamiento y un IRCAN relativamente alto con fundamento en la contaminación generada por los desechos de hogar y la producción agraria e industrial. El interés social hídrico para esta región se fundamenta en las cuencas de los ríos Magdalena y Cauca, así como en las aguas de vertiente de las tres cordilleras del país.

Tabla 1. Indicadores regionales

\begin{tabular}{|c|c|c|c|c|c|c|c|c|}
\hline \multirow[t]{2}{*}{ Regiones } & \multicolumn{8}{|c|}{ Indicadores } \\
\hline & $\begin{array}{l}\text { PRP } \\
(\%)\end{array}$ & $\begin{array}{c}\mathrm{DP} \\
\left(\mathrm{p} / \mathrm{Km}^{2}\right)\end{array}$ & IDH & $\begin{array}{l}\mathrm{AU} \\
(\%)\end{array}$ & $\begin{array}{l}\mathrm{AR} \\
(\%)\end{array}$ & $\begin{array}{l}\text { CDU } \\
(\%)\end{array}$ & IRCA & IRCAN \\
\hline Caribe & 23 & 73 & 0.767 & \multirow{5}{*}{96.9} & \multirow{5}{*}{51.2} & \multirow{5}{*}{89.3} & 13.3 & 64.1 \\
\hline Andina & 56 & 161 & 0.804 & & & & 9.1 & 70.9 \\
\hline Orinoquía & 3 & 5 & 0.792 & & & & 9.2 & 69.2 \\
\hline Pacífica & 16 & 58 & 0.754 & & & & 27.3 & 75.0 \\
\hline Amazonía & 2 & 2 & 0.732 & & & & 20.2 & 75.0 \\
\hline
\end{tabular}

Nota: De acuerdo con PNUD (2009), la calificación del índice de desarrollo humano (IDH) responde a los siguientes rangos: de 0 a 0.499 es bajo; de 5.000 a 0.799 es medio; de 0.800 a 0.899 es alto y $>0.900$ es muy alto.

Fuente: autor con base en Jourablev (2004), IDEAM (2010), Defensoría del Pueblo (2010) y PNUD (2011).

Le sigue, en orden de concentración, la región Caribe, con resultados medios tanto en el IDH como en los índices IRCA e IRCAN; su interés social hídrico se orienta hacia la costa sobre el mar Caribe, la cuenca del río Sinú, la parte baja del río Magdalena y la producción hídrica de la Sierra Nevada.

En el tercer lugar se encuentra la región Pacífica, caracterizada por tener su concentración poblacional hacia el sur, debido a la primacía ejercida por la ciudad de Cali, capital del departamento del Valle. En general, cuenta con índices de riesgo en el consumo de agua tratada y no tratada relativamente altos como respuesta al déficit de saneamiento, especialmente en el departamento de Chocó, y el aporte contaminante de los hogares, la producción agraria e industrial. Su interés social hídrico se orienta hacia la costa sobre el océano Pacífico, los ríos Patía y Atrato y la escorrentía de la vertiente occidental de la cordillera Occidental.

Por último, se encuentran las regiones de la Orinoquía y la Amazonía, con baja concentración poblacional e índices de desarrollo humano; su índice de riesgo en el consumo de agua es medio y alto, respectivamente. El interés social hídrico en la Orinoquía se fundamenta en los ríos provenientes de la región Andina aportantes a la cuenca del río Orinoco; por su parte, la Amazonía reúne los ríos que se enriquecen igualmente con aguas de la región Andina para verterlas en el río Amazonas. 
Además, las evidencias indican una distribución hídrica regional (DHT) amplia para todas las regiones del país entre el inicio y el final del periodo en estudio (2000-2012), pero con ofertas naturales y concentraciones diferenciadas (tabla 2) debido, principalmente, a una sumatoria de eventos tales como la fenomenología climática, la creciente contaminación hídrica nacional con origen en los hogares y los sectores agropecuario e industrial, y al rezago tecnológico en cuanto a la implementación y operación del sistema de saneamiento urbano y rural.

Tabla 2. Distribución y convergencia

\begin{tabular}{|l|c|c|c|c|c|}
\hline \multirow{2}{*}{ Regiones } & \multicolumn{3}{|c|}{ DHT } & \multicolumn{3}{c|}{ DPH } & CC \\
\hline Caribe & 2000 & 2012 & 2000 & 2012 & 2012 \\
\hline Andina & 0.866803 & 0.864538 & 0.179953 & 0.179483 & 0.040946 \\
\hline Orinoquía & 0.578668 & 0.571580 & 0.329072 & 0.313610 & 0.108215 \\
\hline Pacífica & 0.902721 & 0.901063 & 0.025089 & 0.025042 & 0.004939 \\
\hline Amazonía & 0.642280 & 0.636258 & 0.106654 & 0.105654 & 0.032751 \\
\hline
\end{tabular}

La región Andina manifiesta una relación caracterizada por una alta concentración poblacional y la menor oferta hídrica per cápita natural (CG $=0.108215)$, lo cual constituye un evento de vulnerabilidad creciente para su abastecimiento en el futuro (tabla 2 y figura 1). Por su parte, la región Caribe concentra el segundo núcleo de concentración poblacional en Colombia, con una disponibilidad de agua importante, pero, a su vez, con una menor vulnerabilidad $(\mathrm{CC}=0.040946)$, salvo en el departamento de La Guajira, caracterizado por la presencia de un ecosistema desértico.

Figura 1. Mapa radial de convergencia

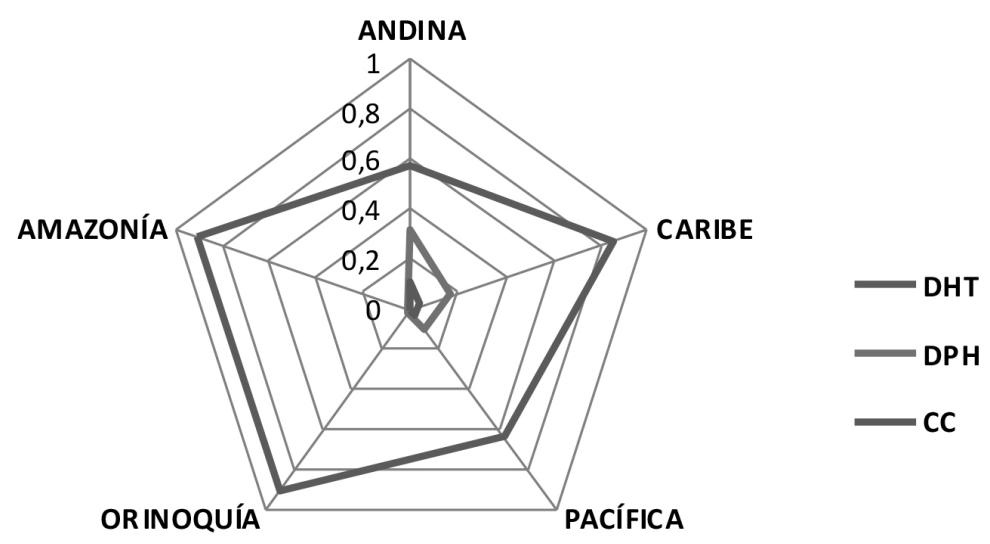

Fuente: autor. 
La región Pacífica cuenta con un régimen pluviométrico de los más altos del mundo, cercano a los 13000 mm-1. Sin embargo, su oferta hídrica per cápita natural ocupa el tercer lugar debido a la diferencia de magnitud de sus vertientes, la temperatura ambiente y la relación proporcional de su volumen poblacional, lo cual conduce a una baja vulnerabilidad $(\mathrm{CG}=0.032751)$. Las regiones de la Orinoquía y la Amazonia presentan vulnerabilidades bajas como respuesta a una reducida concentración poblacional frente a una riqueza hídrica superficial y subterránea de importancia (0.004939 y 0.003297 , respectivamente).

De acuerdo con los planteamientos de Henderson (2000) y la figura 2, los fenómenos de concentración espacial en la región Andina y en la región Caribe manifiestan una desigualdad en la apropiación del territorio nacional, lo cual propicia sesgos económicos e hídricos. Este tipo de comportamiento de la concentración poblacional nacional históricamente afecta el desarrollo de otras regiones al absorber los beneficios económicos del desarrollo, a su vez, repercute en las posibilidades reales de acceso al agua, no solo en cantidad, sino también en calidad, y sustenta estructuralmente trampas de pobreza (CEPAL, 2012).

\section{Discusión}

La ciencia económica reconoce el agua como un bien libre que no debe tener propietarios en particular, e igualmente acepta la intervención del Estado para su regulación y manejo. La evidencia indica una tendencia a consolidar mayores facilidades en el acceso al recurso en el ámbito urbano que rural, sustentada en la preferencia por una relación de uso múltiple población-agua-otros factores, con lo cual se favorece la concentración geográfica, en lugar de una relación unidireccional población-agua solamente, eventualmente con menor productividad de conformidad con los argumentos dados por Pérez, Smits, Benavidez y Vargas (2004).

La oferta hídrica natural en Colombia es superior a su demanda, pero manifiesta características singulares con diferentes patrones regionales. En las tablas y figuras presentadas se observa que la riqueza hídrica por sí sola no es el elemento de atracción primario para concentrar población en una región determinada de Colombia, pues de ser así habría mayor población en la Orinoquía y la Amazonía en contraposición con la región Andina. La respuesta de concentración poblacional obedece a la posibilidad de acceder al agua junto con otros factores, en algunos casos más significativos para la población, como el grado de desarrollo económico regional o urbano, lo cual permite concentrar en un espacio limitado diversas actividades económicas con incrementos de su eficiencia productiva, reducción de costos de transacción (Krugman y Elizondo, 1996), con facilidades para focalizar el gasto público (ONU-HÁBITAT, 2012), lo cual permite construir oportunidades para que la población acceda a ingresos mayores. 
Figura 2. Concentración poblacional y presión hídrica en Colombia

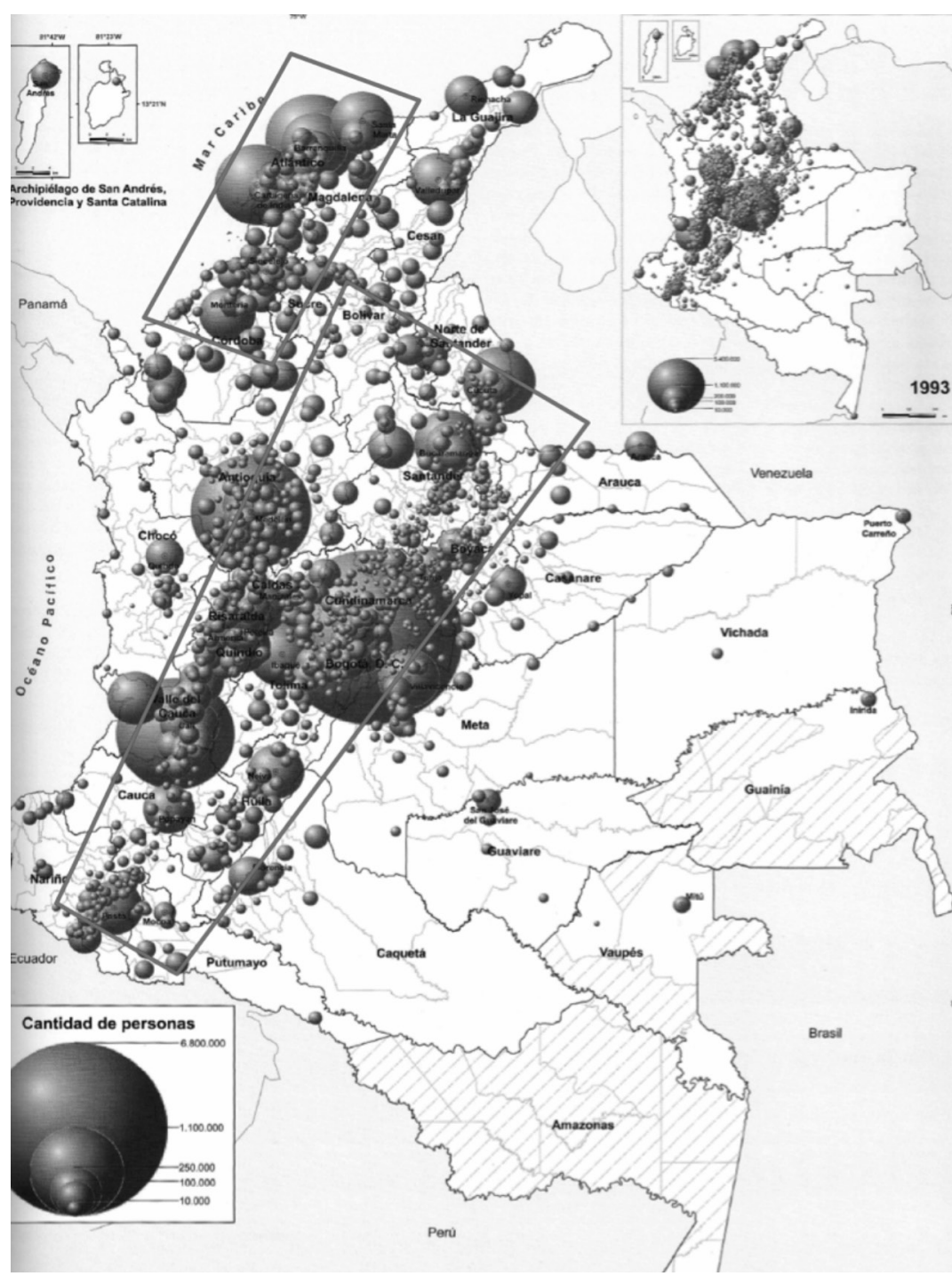

Nota: el mapa permite comparar las condiciones para los años 1993 y 2012.

Fuente: adaptado de DANE (2012).

En consecuencia, el recurso agua conserva su importancia como necesidad, pero pierde su nivel de intensidad en términos económicos. La distorsión de valores sociales en relación con la primacía del agua, especialmente en el ámbito urbano, pasa a un segundo plano. Gana espacio el recurso monetario como elemento fundamental para la supervivencia y por medio del cual es posible acceder al agua, tendencia que ha facilitado la privatización hídrica para su aprovechamiento y comercialización, desdibujando su 
condición de bien común o libre y el derecho ciudadano a su acceso, fenómeno que acentúa inequidades y pobreza, como lo argumenta Shiva (2007).

Contrariamente a la fenomenología de la desconcentración en Colombia expuesta por Montenegro (1996) con base en su condición espacial y la multiplicidad de conglomerados urbanos, Colombia es un país con distribución concentrada de la población, fundamentalmente en dos regiones: la Andina y la Caribe (figura 2). La convergencia poblacional nacional en el futuro se prevé hacia las regiones geográficas con mayor acervo urbano (número de grandes y medianas ciudades) con potencial para sustentar una actividad económica diversificada, creciente y con capacidad de acogida poblacional. Respecto de esta tendencia, Ramírez y Parra-Peña (2013) estiman el coeficiente GINI espacial para Colombia en 0.75, el cual indica la existencia de presiones permanentes sobre el abastecimiento del recurso hídrico para consumo, así como sobre el desarrollo de su infraestructura.

\section{Conclusiones}

Resulta concluyente la presencia de disparidades en la concentración poblacional de base hídrica unida a una situación multipolar urbana, que modifica las facilidades y oportunidades de acceso al recurso agua. La desigualdad hídrica establece la necesidad de una mediación del Estado como política de Estado, no solo en cuanto al ordenamiento territorial y a la conservación del recurso, sino también para garantizar, cuando menos, el suministro mínimo per cápita de agua potable.

En el caso colombiano, el agua por sí sola no es el único factor de referencia para determinar la concentración poblacional regional, ella se articula con oportunidades de supervivencia económica y condiciones de calidad de vida buscadas tanto por los pobladores urbanos como por los rurales. Por lo tanto, se hace necesario establecer una agenda pública para enfrentar el grado de vulnerabilidad que manifiestan la región Andina y la Caribe frente a su oferta hídrica y creciente concentración poblacional. Ello no quiere decir que las demás regiones no requieran de agendas robustas, aplicables y controlables en cuanto a la gestión hídrica bajo condiciones de cambio climático. 


\section{Referencias}

Botiva, Á., Groot, A. M., Herrera, L. y Mora, S. (1989). Colombia prehispánica. Regiones arqueológicas. Bogotá: Instituto Colombiano de Antropología.

CEPAL. (2009). Economía y territorio en América Latina y el Caribe. Santiago de Chile: Autor.

CEPAL. (2012). Población, territorio y desarrollo sostenible. Santiago de Chile: Naciones Unidas.

Correa, G. (2011). Economía ambiental en la prehistoria colombiana. Gestión y Sociedad, $4(1), 117-128$.

Cuervo, L. (2004). Desarrollo económico y primacía urbana en América Latina. En, El rostro urbano de América Latina (pp. 77-114). Buenos Aires: CLACSO.

DANE. (2005). Censo general a nivel nacional. Bogotá: Autor.

DANE. (2012). Atlas estadístico de Colombia (Tomo I). Bogotá: DANE.

Defensoría del Pueblo. (noviembre de 2011). Diagnóstico de la calidad del agua para consumo humano. Año 2010. Bogotá: Defensoría del Pueblo.

Defensoría del Pueblo. (noviembre de 2011). Emergencia en Colombia por el fenómeno de La Niña 2010-2011. Bogotá: Defensoría del Pueblo.

Guevara, D. (2012). Desafios, vicisitudes y quimeras de la población desplazada ante la pobreza. Bogotá: FICA.

Hardin, G. (1995). La tragedia de los comunes. Gaceta Ecológica, 162(37), 1243-1248. Recuperado de https://goo.gl/bOFjij

Henderson, V. (abril de 2000). How urban concentration affects economic growth. Policy Research Working Paper (n.o 2326). Washington: Banco Mundial.

IDEAM. (2010). Estudio nacional de agua. Bogotá: IDEAM.

IGAC. (2011). Geografia de Colombia. Bogotá: Imprenta Nacional de Colombia - IGAC.

Jourablev, A. (2004). Los servicios de agua potable y saneamiento en el umbral del siglo XXI. Santiago de Chile: CEPAL.

Krugman, P. (1991). Geography and Trade. Massachusetts: MIT Press. 
Krugman, P., \& Elizondo, R. (1996). Trade Policy and the Third World Metrópolis. Journal of Development Economics, 49, 137-150.

Lombardo, J. D. (2007). Paradigmas urbanos. Conceptos e ideas que sostienen la ciudad actual. Buenos Aires: Universidad Nacional General Sarmiento.

Montenegro, S. (1996). El papel de las regiones para la estabilidad macroeconómica de Colombia. Revista Desarrollo y Sociedad, 38, 63-104.

Olson, M. (1985). La lógica de la acción colectiva. En, Auge y decadencia de las naciones (pp. 32- 55). Barcelona: Ariel.

ONU. (2012). La sostenibilidad del desarrollo a 20 años de la Cumbre de la Tierra. Santiago de Chile: CEPAL.

ONU-HÁBITAT. (2012). Estado de las ciudades de América Latina y el Caribe. Río de Janeiro: ONU.

Ostrom, E. (2009). El gobierno de los bienes comunes. La evolución de las instituciones de acción colectiva. México: Fondo de Cultura Económica.

Pérez, M., Smits, S., Benavidez, A. y Vargas, S. (2004). Multiple uso of water, livelihoods and poverty in Colombia: a case study from the Ambichinte micro-catchment. En P. Moriarty, J. Butterworth, B. Van Koppen (Eds.), Beyond Domestic: Case studies on poverty and productive uses of water at the household level (pp. 75- 93). Holland: IRC.

PNUD. (2006). Más allá de la escasez: poder, pobreza y la crisis mundial del agua. Madrid: Mundiprensa.

PNUD. (2009). Superando barreras: movilidady desarrollo humanos. Madrid: Mundi Prensa.

PNUD. (2011). Informe Nacional del Desarrollo Humano. Bogotá: INDH-PNUD.

Ramírez, J. y Parra-Peña, R. (2013). Metrópolis de Colombia, aglomeraciones y desarrollo. Bogotá: CEPAL.

Rojas, S. (2010). Análisis espacial y patrones de asentamientos en el bajo río San Jorge. Boletín de Antropología, 41(24), 283-305. Recuperado de https://goo.gl/vQK8Ef

Sánchez-Triana, E. (2007). Un modelo particular de gestión ambiental. En E. SánchezTriana, K. Ahmed, \& Y. Awe, Prioridades ambientales para la reducción de la pobreza en Colombia. Un análisis ambiental del país para Colombia (pp. 25-44). Bogotá: Banco Mundial y Mayol Ediciones S. A.

Shiva, V. (2007). Las guerras del agua. México: Siglo XXI Editores. 
